\title{
The Natural History of High-Grade Partial Thickness Rotator Cuff Tears: The Conversion Rate to Full Thickness Tears and Affecting Factors
}

\author{
Joo Han Oh, MD, Ye Hyun Lee, MD*, Tae Ho Lee, MD*, Seok In Jang, MD*, Jieun Kwon, MD* \\ Department of Orthopedic Surgery, Seoul National University Hospital, Seoul National University College of Medicine, Seoul, \\ ${ }^{*}$ Department of Orthopedic Surgery, National Police Hospital, Seoul, Korea
}

Background: Information regarding the progression of high-grade partial thickness rotator cuff tears (PTRCTs) is scarce. We aimed to assess the clinical outcome and the conversion rate to full thickness tears in patients with high-grade PTRCTs who underwent nonoperative treatment and to determine the factors associated with tear progression.

Methods: A total of 52 patients with high-grade PTRCTs, which were detected by magnetic resonance imaging or ultrasonography (USG), were treated conservatively between 2010 and 2017. They were followed up with USG at 6- to 12-month intervals for a mean of 34 months (range, 12-105 months). The average patient age was 57 years (range, 34-70 years), and 34 patients were women. Age, sex, body mass index, arm dominance, symptom duration, subscapularis tendon involvement, tear location, and trauma history were compared between patients with and without conversion to full thickness tears.

Results: A substantial percentage of high-grade PTRCTs progressed to full thickness tears (16/52, 30.8\%). According to KaplanMeier analysis, the full thickness conversion rate was $30.8 \%$ at 3 years and $64 \%$ at 4 years. The full thickness conversion rate was higher in patients with subscapularis tendon involvement $(p=0.012)$.

Conclusions: A considerably large proportion of high-grade PTRCTs progressed to full thickness tears. Therefore, regular monitoring of tear progression should be considered after conservative treatment of high-grade PTRCTs, particularly in patients with subscapularis tendon involvement.

Keywords: Shoulder, Rotator cuff, Subscapularis

Partial thickness rotator cuff tears (PTRCTs) are a common pathology that causes shoulder pain and disability. Previous cadaveric and imaging studies reported that the prevalence of PTRCTs ranges from $13 \%$ to $32 \% .{ }^{1-5)}$ Despite its high prevalence, the information regarding PTRCTs is scarce. Only a few studies have described the natural history of PTRCTs, and the studies published on this topic have reported inconsistent results. Yamanaka and Mat-

Received December 9, 2019; Accepted March 22, 2020

Correspondence to: Jieun Kwon, MD

Department of Orthopedic Surgery, National Police Hospital, 123 Songiro, Songpa-gu, Seoul 05715, Korea

Tel: +82-2-3400-1333, Fax: +82-2-3400-1249

E-mail: toast8315@gmail.com sumoto reported that $80 \%$ of arthroscopically monitored PTRCTs eventually enlarged or progressed to full thickness tears over 2 years. ${ }^{6}$ Mall et al. ${ }^{7)}$ monitored 30 subjects with asymptomatic PTRCTs and found that 10 tears became symptomatic; of these 10 tears, $40 \%$ progressed to full thickness rotator cuff tears. In contrast, Maman et al. ${ }^{8}$ managed 26 symptomatic PTRCTs nonoperatively and found that only $8 \%$ of the cases progressed in size on magnetic resonance imaging (MRI).

Although various factors may affect the decision to perform surgery, ${ }^{3,9)}$ in general, the failure of nonoperative treatment and tearing of $>50 \%$ of the tendon thickness are considered as important factors in determining surgical intervention. ${ }^{5)}$ Historically, Ellman ${ }^{9)}$ classified PTRCTs based on the percentage of tendon thickness involved in 
Oh et al. Natural History of High-Grade Partial Thickness Rotator Cuff Tears

Clinics in Orthopedic Surgery • Vol. 12, No. 4, $2020 \bullet$ www.ecios.org

the tear (grade $1,<25 \%$; grade $2,<25 \%-50 \%$; and grade 3 , $>50 \%)$. In a recent biomechanical study, Mazzocca et al. ${ }^{10)}$ found that PTRCTs involving $>50 \%$ of the tendon thickness (high-grade PTRCTs) increased the strain on the remaining intact portion of the tendon, as compared with an intact tendon. Although several studies have shown good clinical results in patients who were surgically treated for high-grade PTRCTs, ${ }^{11-13)}$ the natural history of high-grade PTRCTs remains unclear and the operative treatment of high-grade PTRCTs is still controversial. Further understanding of the natural history of high-grade PTRCTs could help identify patients at risk for tear progression and be expected to help orthopedic surgeons determine their therapeutic decisions.

In the present study, we aimed to assess the clinical outcomes and the conversion rate to full thickness rotator cuff tears in patients with high-grade PTRCTs who were treated nonoperatively and to determine the factors associated with tear progression. The hypothesis of this study was that a larger-than-expected proportion of PTRCTs would progress to full thickness tears.

\section{METHODS}

\section{Cohort}

Institutional Review Board of Seoul National University Bundang Hospital approved this study (IRB No. B-1512328-110). Clinical records of patients who had symptomatic high-grade PTRCTs, diagnosed using MRI or ultrasonography (USG) between 2010 and 2017, were retrospectively analyzed. The patients received treatment with physiotherapy, nonsteroidal anti-inflammatory drugs, and subacromial or intra-articular steroid injections and were followed up clinically along with USG examinations at 6-12-month intervals. Inclusion criteria were (1) supraspinatus tears involving $>50 \%$ of the tendon thickness, regardless of the presence of other tendon tears, ${ }^{9)}$ (2) patients with a follow-up of at least 12 months, (3) patients who underwent 2 or more USG examinations, and (4) no traumatic episode throughout the study period. Exclusion criteria were (1) other rotator cuff partial tears not including supraspinatus tears, (2) patients who underwent surgery before 12 months, (3) history of previous surgery in the affected shoulder, and (4) history of fracture in the affected shoulder. During the study period, a total of 105 patients were identified to have symptomatic high-grade PTRCTs and undergo 2 or more USG examinations. Fifty-three of the 105 patients were excluded for the following reasons: 37 were not followed up for more than 12 months, 10 underwent surgery due to pain aggravation before 12 months, 3 underwent surgery due to full thickness conversion before 12 months, 3 had previous surgery on the affected shoulder. Finally, a total of 52 patients, including 18 male (34.6\%) and 34 female (65.4\%) patients, were enrolled. The average age of the included patients was 57 years (range, 34-70 years). There were 30 articular- and 22 bursal-side partial thickness tears. The minimum follow-up duration was 12 months, and the mean follow-up duration was 34 months (standard deviation, 19.3 months; range, 12-105 months).

\section{Clinical Assessment}

At the initial diagnosis, active range of motion (ROM) measurements were performed by fellowship trained orthopedic surgeons using a standardized method involving a goniometer ${ }^{14,15)}$ Functional assessments were performed using the Constant-Murley score and simple shoulder test. The follow-up examinations, performed at 6- to 12-month intervals, involved all the components of the initial clinical assessment, including USG.

Age, sex, body mass index (BMI), symptom duration, involved side, subscapularis tendon involvement, tear location (articular-side or bursal-side PTRCTs), and trauma history were compared between patients with and without conversion to full thickness tears.

\section{Imaging}

All the patients with suspected rotator cuff tears were recommended to undergo USG for further evaluation at the initial visit except for some patients who had already undergone MRI at other clinics. USG was performed by trained musculoskeletal radiologists using a standardized method (iU22 ultrasound scanner; Philips Healthcare, Bothell, WA, USA). A high-grade PTRCT was diagnosed in cases with a flattening of the bursal side involving $>50 \%$ of the tendon thickness (a bursal-side partial thickness tear) or a distinct hypoechoic or mixed hyperechoic and hypoechoic defect involving $>50 \%$ of the tendon thickness at the deep articular side of the tendon (an articular-side partial thickness tear). ${ }^{16,17)}$ Full thickness conversion was defined as the complete disruption of tendon continuity at the normal tendon footprint on the greater tuberosity. In the present study, a full thickness conversion rather than an increase in tear size was only regarded as meaningful enlargement.

\section{Statistical Analysis}

All statistical analyses were performed with IBM SPSS ver. 20.0 (IBM Corp., Armonk, NY, USA), and p-values $<0.05$ were considered statistically significant. Student $t$-test, paired $t$-test, or Mann-Whitney test was used for the comparison of continuous variables such as ROM, functional 
Oh et al. Natural History of High-Grade Partial Thickness Rotator Cuff Tears

Clinics in Orthopedic Surgery • Vol. 12, No. 4, $2020 \bullet$ www.ecios.org

score, age, BMI, and symptom duration. Moreover, the chisquare test or Fisher exact test was used for comparison of categorical variables including sex, involved side, subscapularis tendon involvement, trauma history, and tear location. A Kaplan-Meier plot was used to determine the full thickness conversion rate.

\section{RESULTS}

At the most recent visit, the partial thickness tears in 16 patients (30.8\%) had progressed to full thickness tears, and the conversion was noted within 3 years in $10(62.5 \%)$ of these 16 cases. Six patients (37.5\%) underwent arthroscopic rotator cuff repair surgery, whereas the remaining 10 patients were managed conservatively due to old age or low activity demand. The median time to full thickness conversion was $37.6 \pm 20.6$ months (range, 14-105 months) and the median observation time of the no change group was $30.8 \pm 16.8$ months (range, 12-69 months). The average number of examinations was $4.8 \pm 1.9$ (range, 2-8) in the full thickness conversion group and $4.1 \pm 1.1$ (range, $3-7)$ in the no change group. There was no significant difference in observation time $(p=0.218)$ and frequency of examination ( $p=0.177$ ) between 2 groups. The KaplanMeier plotting, which described the progress of partial thickness tears with full thickness conversion as the endpoint, indicated a full thickness conversion rate of $4.5 \%$ at 1 year, $23.2 \%$ at 2 years, $33.1 \%$ at 3 years, and $64.0 \%$ at 4 years (Fig. 1).

The Constant score improved from 71.6 to 78.8 in patients with full thickness conversion and from 69.4 to 81.0 in those without $(p=0.231)$. The simple shoulder test improved from 7.6 to 8.7 in patients with full thickness conversion and from 6.8 to 8.5 in those without $(p=0.320)$. There

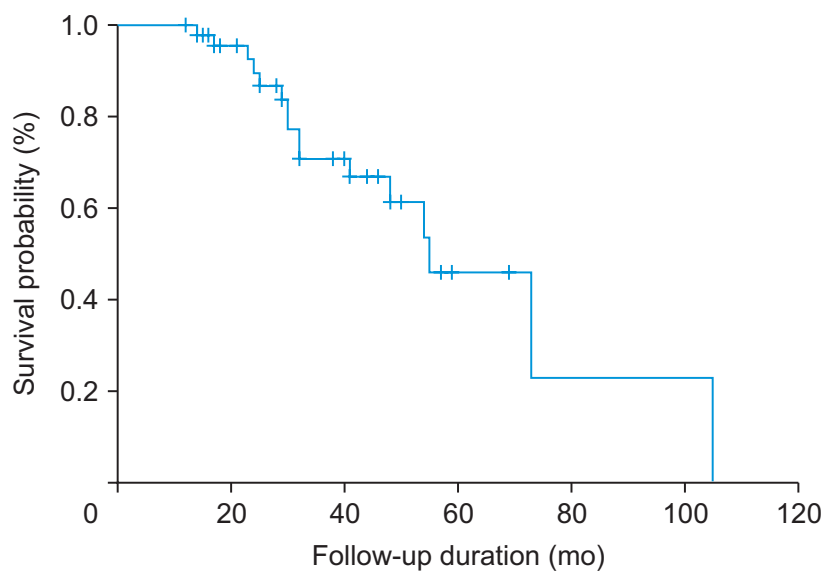

Fig. 1. Kaplan-Meier survival curve for full thickness conversion. was no difference in the functional results between patients with and without full thickness conversion (Table 1).

The full thickness conversion rate was higher in patients with subscapularis tendon involvement $(p=0.012)$. Moreover, partial thickness tears of the subscapularis tendon were observed in $50.0 \%$ of patients with full thickness conversion and in $13.9 \%$ of patients with no progression. None of the patients exhibited a full thickness tear of the subscapularis tendon. In addition, there was no association between the full thickness conversion rate and sex $(p=0.367)$, age $(p=0.780)$, BMI $(p=0.402)$, symptom duration $(p=0.194)$, involved side $(p=0.229)$, and presence of trauma history at the time of onset $(p=0.140)$. In addition, the tear location (articular or bursal side) did not affect the full thickness conversion rate $(p=0.888)$ (Table 2).

\section{DISCUSSION}

In the present study, a large proportion of cases with supraspinatus high-grade partial thickness tears (30.8\%) progressed to full thickness tears, and the full thickness conversion rate was found to be correlated with the presence of partial thickness subscapularis tears $(p=0.012)$. Several studies have been reported on the natural course of full thickness rotator cuff tears. However, to our knowledge, this is the first study that specifically focuses on the natural course of high-grade PTRCTs.

The development of imaging modalities such as MRI and USG has enabled the detection of an increasing number of PTRCTs. ${ }^{18)}$ Despite its high prevalence, ${ }^{1-5)}$ the data on the natural history of PTRCTs are relatively scarce. Maman et al. ${ }^{8)}$ reported that more than half (52\%) of the cases with full thickness tears exhibited an increase in size, as compared to only $8 \%$ of the PTRCTs. Mall et al. ${ }^{7)}$ showed that $40 \%$ of the PTRCTs had progressed to full thickness tears. Moosmayer et al. ${ }^{19)}$ indicated that a considerable percentage of asymptomatic rotator cuff tears became symptomatic and showed anatomical deterioration. Recently, Keener et al. ${ }^{20)}$ evaluated the survivorship in cases of asymptomatic degenerative rotator cuff tears: enlargement was observed in $61 \%$ of full thickness tear cases and $44 \%$ of the partial thickness tears were associated with the onset of new pain. These studies had a small number of patients with PTRCTs and did not assess the factors associated with tear progression.

The present study indicated a high risk of full thickness conversion over a relatively short time. At a median duration of 31 months, $30.8 \%$ of high-grade PTRCTs exhibited conversion to full thickness tears. The full thick- 
Oh et al. Natural History of High-Grade Partial Thickness Rotator Cuff Tears

Clinics in Orthopedic Surgery • Vol. 12, No. 4, $2020 \bullet$ www.ecios.org

Table 1. Functional Assessment between the FT Conversion Group and No Progression Group

\begin{tabular}{|c|c|c|c|}
\hline Variable & FT conversion & No progression & $p$-value* \\
\hline Forward flexion & & & 0.433 \\
\hline Initial & $152 \pm 19.9$ & $148.2 \pm 17.6$ & \\
\hline Last FU & $161.3 \pm 11.0$ & $161.7 \pm 8.0$ & \\
\hline Change & $8.8 \pm 21.2$ & $13.5 \pm 15.9$ & \\
\hline Internal rotation at back & & & 0.800 \\
\hline Initial & $10.2 \pm 4.8$ & $10.0 \pm 3.8$ & \\
\hline Last FU & $8.3 \pm 2.6$ & $7.8 \pm 2.2$ & \\
\hline Change & $1.9 \pm 4.6$ & $2.2 \pm 2.8$ & \\
\hline Constant-Murley score & & & 0.231 \\
\hline Initial & $71.6 \pm 11.4$ & $69.4 \pm 10.6$ & \\
\hline Last FU & $78.8 \pm 8.2$ & $81.0 \pm 6.5$ & \\
\hline Change & $7.2 \pm 12.3$ & $11.6 \pm 10.8$ & \\
\hline Simple shoulder test & & & 0.320 \\
\hline Initial & $7.6 \pm 1.8$ & $6.8 \pm 1.3$ & \\
\hline Last FU & $8.7 \pm 1.6$ & $8.5 \pm 1.0$ & \\
\hline Change & $1.1 \pm 2.4$ & $1.8 \pm 1.5$ & \\
\hline
\end{tabular}

Values are presented as mean \pm standard deviation.

FT: full thickness, FU: follow-up.

${ }^{*}$ Statistically significant difference between groups $(p<0.05)$.

Table 2. Association between Clinical Factors and Tear Progression

\begin{tabular}{|c|c|c|c|}
\hline Variable & $\begin{array}{l}\text { Full thickness } \\
\text { conversion }\end{array}$ & No change & $p$-value* \\
\hline Sex (male : female) & $7: 9$ & $11: 25$ & 0.367 \\
\hline Age (yr) & $56.1 \pm 7.6$ & $56.8 \pm 7.9$ & 0.780 \\
\hline Body mass index $\left(\mathrm{kg} / \mathrm{m}^{2}\right)$ & $23.9 \pm 1.6$ & $24.5 \pm 3.3$ & 0.402 \\
\hline Symptom duration (mo) & $39.5 \pm 23.4$ & $30.8 \pm 16.8$ & 0.194 \\
\hline Involved side (Rt : Lt) & $11: 5$ & $17: 19$ & 0.229 \\
\hline SSC involvement (+ :-) & $8: 8$ & $31: 5$ & 0.012 \\
\hline $\begin{array}{l}\text { Tear location } \\
\text { (articular : bursal) }\end{array}$ & $9: 7$ & $21: 15$ & 0.888 \\
\hline Trauma history (+ : -) & $1: 15$ & $10: 26$ & 0.140 \\
\hline
\end{tabular}

Values are presented as mean \pm standard deviation.

Rt: right, Lt: left, SSC: subscapularis tendon.

*Statistically significant difference between groups $(p<0.05)$. ness conversion rate in the present study is slightly higher than the rates in previous studies on nonoperatively treated PTRCTs. Maman et al. ${ }^{8)}$ reported that only $8 \%$ of PTRCTs exhibited conversion to full thickness tears, and Keener et al. ${ }^{20)}$ observed that approximately $20 \%$ of PTRCTs progressed to full thickness tears. Although Mall et $\mathrm{al}^{7}{ }^{7}$ observed a higher rate of full thickness conversion (40\%), they included only 10 subjects. One reason for the high incidence of full thickness conversion in the present study, as compared to other studies, might be related to the inclusion of only high-grade PTRCTs. In a study by Matthewson et al., ${ }^{5)}$ only $8 \%$ of 37 PTRCTs progressed to full thickness tears at an average follow-up of 53 months after nonoperative treatment, whereas among the patients with tears $>50 \%$ thickness, tear progression was observed in $55 \%$ of cases. In a biomechanical study, Mazzocca et al. ${ }^{10)}$ found that compared with an intact tendon, PTRCTs involving $>50 \%$ of the tendon thickness increased the strain on the remaining intact portion of the tendon. Thus, high-grade PTRCTs have a low potential for spontaneous healing and high risk of full thickness conversion. In the present study, we confirmed that a considerably large per- 
Oh et al. Natural History of High-Grade Partial Thickness Rotator Cuff Tears

Clinics in Orthopedic Surgery • Vol. 12, No. 4, $2020 \bullet$ www.ecios.org

centage of high-grade PTRCTs progressed to full thickness tears despite the improvement in functional scores. Hence, we believe that high-grade PTRCTs should be managed differently from low-grade PTRCTs and monitored regularly, regardless of the presence of symptoms.

The present study showed a statistically significant association between tear progression and subscapularis tendon involvement $(p=0.012)$. A subscapularis partial thickness tear was observed in $50 \%$ of patients in the full thickness conversion group and in $13.6 \%$ of patients in the no progression group. Although force couple theory is commonly applied to large rotator cuff tears, the biomechanical importance of the subscapularis tendon has been emphasized in this theory proposed by Burkhart ${ }^{21)}$ Several recent studies have investigated the biomechanical role in subscapularis tears, and some of these studies have described the effect of partial thickness subscapularis tears. Marquardt et el. ${ }^{22)}$ reported that the intra-articular portion of the subscapularis tendon increased glenohumeral translation in all directions. In addition, Yoo et al. ${ }^{23)}$ showed an increase in external rotation and superior shift in the humeral head apex position in cases with subscapularis partial thickness tears. Such biomechanical changes in subscapularis partial thickness tears may increase the strain on the supraspinatus tendon, which could then be vulnerable to conversion to full thickness tears. Jost et al. ${ }^{24)}$ reported that isolated supraspinatus tears might experience less severe degeneration over time, and this finding was also consistent with the results of this study.

The present study has certain limitations. The first main limitation is related to the retrospective design of this study. Some patients diagnosed as having high-grade PTRCTs at the initial diagnosis were excluded from the study due to follow-up loss. As described in the materials and methods section, during the study period, a total of 105 patients with symptomatic high-grade PTRCTs were identified and underwent 2 or more USG examinations. Among them, 37 patients were not followed up for more than 12 months. It is likely that either these patients had improvement and did not return for follow-up or that the nonoperative treatment performed at our clinic failed and they received treatment at another clinic. Moreover, the long study period (between 2010 and 2017) could have affected the results. Second, different imaging modalities (MRI and USG) were used for evaluation. Some patients underwent MRI before presenting to our clinic. This could have partially affected the assessment of the progression of tear size at the final follow-up. However, the number of patients who had undergone MRI at another clinic was small (7 patients, $13.5 \%)$, and all these patients exhibited a similar tear size at the follow-up after the initial diagnosis. Hence, we do not believe that the difference in imaging modality for evaluation markedly affected the outcome of tear progression. Third, progression of horizontal tears was not considered in this study. Several previous studies showed that the diagnostic performance of USG and MRI was similar for detecting PTRCTs. ${ }^{16,25)}$ However, both USG and MRI had poor sensitivity for identifying the size of PTRCTs; USG correctly predicted the width of $54 \%$ and length of $87 \%$ of PTRCTs and MRI correctly predicted the width of $75 \%$ and length of $75 \%$ of PTRCTs when compared with arthroscopic findings. ${ }^{16)}$ Moreover, subscapularis tears could be more difficult to evaluate with these imaging modalities. Therefore, in the present study, only full thickness conversion was regarded as meaningful enlargement rather than the presence of an increase in tear size. Fourth, the correct progression time could not be identified because of the follow-up interval. If a patient showed no progression at the 12-month follow-up and showed progression at 24 months, the progression time of this patient was considered as 24 months. On the other hand, if a patient showed no progression at 12-month follow-up and showed progression at 18 months, the progression time of this patient was considered as 18 months. However, both patients could have progression at the same 14 months after the initial visit. Finally, there are some factors that should be considered when interpreting the results. According to the exclusion criteria, 13 patients were excluded from the study: 10 patients who had surgery due to aggravation of pain and 3 patients who had progression to a full thickness tear before the minimum follow-up of 12 months. There was no significant clinical difference between the full thickness conversion and non-conversion groups. It might be due to this exclusion. However, they did not meet the minimum follow-up period; therefore, it was thought be to suitable to exclude them from the data analysis in view of data management. Additionally, there is no agreement that patients with high-grade PTRCTs should undergo surgery if there was no response to the conservative treatment. We thought that it was important to confirm the progression of the remaining high-grade PTRCTs, which was the inclusion criteria of this study. Despite such limitations, the present study is the first study to investigate the natural history of high-grade PTRCTs and the factors associated with tear progression. Our findings could be useful for orthopedic surgeons to determine the progression of high-grade PTRCTs and guide them in patient management.

In conclusion, in the present study of nonoperatively treated high-grade PTRCTs, a considerably large propor- 
Oh et al. Natural History of High-Grade Partial Thickness Rotator Cuff Tears

Clinics in Orthopedic Surgery • Vol. 12, No. 4, $2020 \bullet$ www.ecios.org

tion of cases progressed to full thickness tears. Therefore, regular monitoring of tear progression is advised after conservative treatment of high-grade PTRCTs, especially in patients with subscapularis tendon involvement.

\section{CONFLICT OF INTEREST}

No potential conflict of interest relevant to this article was reported.

\section{REFERENCES}

1. Fukuda H. Partial-thickness rotator cuff tears: a modern view on Codman's classic. J Shoulder Elbow Surg. 2000;9(2): 163-8.

2. Sano H, Ishii H, Trudel G, Uhthoff HK. Histologic evidence of degeneration at the insertion of 3 rotator cuff tendons: a comparative study with human cadaveric shoulders. J Shoulder Elbow Surg. 1999;8(6):574-9.

3. Lohr JF, Uhthoff HK. The microvascular pattern of the supraspinatus tendon. Clin Orthop Relat Res. 1990;(254):35-8.

4. Sher JS, Uribe JW, Posada A, Murphy BJ, Zlatkin MB. Abnormal findings on magnetic resonance images of asymptomatic shoulders. J Bone Joint Surg Am. 1995;77(1):10-5.

5. Matthewson G, Beach CJ, Nelson AA, et al. Partial thickness rotator cuff tears: current concepts. Adv Orthop. 2015;2015: 458786.

6. Yamanaka K, Matsumoto $\mathrm{T}$. The joint side tear of the rotator cuff: a followup study by arthrography. Clin Orthop Relat Res. 1994;(304):68-73.

7. Mall NA, Kim HM, Keener JD, et al. Symptomatic progression of asymptomatic rotator cuff tears: a prospective study of clinical and sonographic variables. J Bone Joint Surg Am. 2010;92(16):2623-33.

8. Maman E, Harris C, White L, Tomlinson G, Shashank M, Boynton E. Outcome of nonoperative treatment of symptomatic rotator cuff tears monitored by magnetic resonance imaging. J Bone Joint Surg Am. 2009;91(8):1898-906.

9. Ellman H. Diagnosis and treatment of incomplete rotator cuff tears. Clin Orthop Relat Res. 1990;(254):64-74.

10. Mazzocca AD, Rincon LM, O'Connor RW, et al. Intra-articular partial-thickness rotator cuff tears: analysis of injured and repaired strain behavior. Am J Sports Med. 2008;36(1):110-6.

11. Fukuda $\mathrm{H}$. The management of partial-thickness tears of the rotator cuff. J Bone Joint Surg Br. 2003;85(1):3-11.

12. Kartus J, Kartus C, Rostgard-Christensen L, Sernert N, Read J, Perko M. Long-term clinical and ultrasound evaluation after arthroscopic acromioplasty in patients with partial rotator cuff tears. Arthroscopy. 2006;22(1):44-9.

13. Kamath G, Galatz LM, Keener JD, Teefey S, Middleton W, Yamaguchi K. Tendon integrity and functional outcome after arthroscopic repair of high-grade partial-thickness su- praspinatus tears. J Bone Joint Surg Am. 2009;91(5):1055-62.

14. Park JS, Park HJ, Kim SH, Oh JH. Prognostic factors affecting rotator cuff healing after arthroscopic repair in small to medium-sized Tears. Am J Sports Med. 2015;43(10):2386-92.

15. Chung SW, Kim JY, Kim MH, Kim SH, Oh JH. Arthroscopic repair of massive rotator cuff tears: outcome and analysis of factors associated with healing failure or poor postoperative function. Am J Sports Med. 2013;41(7):1674-83.

16. Teefey SA, Rubin DA, Middleton WD, Hildebolt CF, Leibold RA, Yamaguchi K. Detection and quantification of rotator cuff tears: comparison of ultrasonographic, magnetic resonance imaging, and arthroscopic findings in seventy-one consecutive cases. J Bone Joint Surg Am. 2004;86(4):708-16.

17. Teefey SA, Hasan SA, Middleton WD, Patel M, Wright RW, Yamaguchi K. Ultrasonography of the rotator cuff: a comparison of ultrasonographic and arthroscopic findings in one hundred consecutive cases. J Bone Joint Surg Am. 2000;82(4):498-504.

18. Xiao J, Cui GQ. Clinical and magnetic resonance imaging results of arthroscopic repair of intratendinous partial-thickness rotator cuff tears. Chin Med J (Engl). 2015;128(11):1496-501.

19. Moosmayer S, Tariq R, Stiris M, Smith HJ. The natural history of asymptomatic rotator cuff tears: a three-year followup of fifty cases. J Bone Joint Surg Am. 2013;95(14):1249-55.

20. Keener JD, Galatz LM, Teefey SA, et al. A prospective evaluation of survivorship of asymptomatic degenerative rotator cuff tears. J Bone Joint Surg Am. 2015;97(2):89-98.

21. Burkhart SS. Arthroscopic treatment of massive rotator cuff tears: clinical results and biomechanical rationale. Clin Orthop Relat Res. 1991;(267):45-56.

22. Marquardt B, Garmann S, Hurschler C, et al. The influence of arthroscopic subscapularis tendon and anterior capsular release on glenohumeral translation: a biomechanical model. J Shoulder Elbow Surg. 2006;15(4):502-8.

23. Yoo JC, McGarry MH, Jun BJ, Scott J, Lee TQ. The influence of partial subscapularis tendon tears combined with supraspinatus tendon tears. J Shoulder Elbow Surg. 2014; 23(6):902-8.

24. Jost B, Zumstein M, Pfirrmann CW, Gerber C. Long-term 
Oh et al. Natural History of High-Grade Partial Thickness Rotator Cuff Tears

Clinics in Orthopedic Surgery • Vol. 12, No. 4, $2020 \bullet$ www.ecios.org

outcome after structural failure of rotator cuff repairs. J Bone Joint Surg Am. 2006;88(3):472-9.

25. Lenza M, Buchbinder R, Takwoingi Y, Johnston RV, Hanchard NC, Faloppa F. Magnetic resonance imaging, magnetic resonance arthrography and ultrasonography for assessing rotator cuff tears in people with shoulder pain for whom surgery is being considered. Cochrane Database Syst Rev. 2013;2013(9):CD009020. 